\title{
Percepciones de docentes en formación de pedagogía básica sobre educar en contextos vulnerables
}

Yancovic-Allen, Macarena; Escobar-González, Sebastián

Percepciones de docentes en formación de pedagogía básica sobre educar en contextos vulnerables

Revista Educación, vol. 46, núm. 1, 2022

Universidad de Costa Rica, Costa Rica

Disponible en: https://www.redalyc.org/articulo.oa?id=44068165008

DOl: https://doi.org/10.15517/revedu.v46i1.43787

\section{(c) $(1) \Theta$}

Esta obra está bajo una Licencia Creative Commons Atribución-NoComercial-SinDerivar 3.0 Internacional. 


\title{
Percepciones de docentes en formación de pedagogía básica sobre educar en contextos vulnerables
}

\author{
Perception of Preservice Elementary Teachers Regarding Teaching Vulnerable Population Groups
}

Macarena Yancovic-Allen

Universidad Finis Terrae, Chile

myancovic@uft.cl

(D) https://orcid.org/0000-0002-3179-817X

Sebastián Escobar-González

Universidad Finis Terrae, Chile

sescobar@uft.cl

(D) https://orcid.org/0000-0003-2786-1487

\author{
DOI: https://doi.org/10.15517/revedu.v46i1.43787 \\ Redalyc: https://www.redalyc.org/articulo.oa? \\ $\mathrm{id}=44068165008$
}

Recepción: 16 Septiembre 2020

Aprobación: 23 Diciembre 2020

\section{Resumen:}

El 50 \% de jóvenes de América Latina está en condiciones de vulnerabilidad. En Chile, a nivel escolar, esta realidad se eleva hasta el 76,4\%. Por este motivo, la presente investigación busca indagar en las percepciones de docentes en formación de enseñanza primaria con respecto al trabajo educativo realizado en sus prácticas pedagógicas en contextos vulnerables. A través de un diseño cualitativo, se realizaron entrevistas semiestructuradas a catorce docentes en formación de una universidad privada de la capital chilena. Una vez transcritas las entrevistas, se llevó a cabo un análisis temático en donde se leyeron reiteradamente las transcripciones para identificar códigos iniciales -que permitieron la búsqueda de temas-, su revisión y final definición. Los resultados identifican dos temas principales que hacen relación a las tensiones y desafíos que percibió el estudiantado de pedagogía en la práctica pedagógica realizada en estos contextos. Cada tema está compuesto de cuatro códigos, los cuales se pueden comprender y profundizar desde diversas aristas. Entre las tensiones se destaca el trabajo en contextos de violencia, la relación que se establece entre docentes y estudiantes, el currículum oficial y el currículum implementado, y la desesperanza aprendida experimentada por el grupo de estudiantes. Con respecto a los desafíos, se destaca la interacción con el alumnado, la promoción de valores en la sala de clases, el manejo de las emociones y, finalmente, la frustración en la lección. A través de los resultados, esta investigación busca entregar insumos a los programas de pedagogía, con el fin de fortalecer el proceso de formación docente al entregar herramientas que les permitan desenvolverse en estos contextos. A la vez, busca ser un aporte para docentes que trabajan en estos contextos, al profundizar en la comprensión de este fenómeno.

Palabras Clave: Enseñanza primaria, Formación de docentes, Ambiente educacional, Contextos vulnerables.

\section{Abstract:}

Approximately 50\% of Latin America's youth pertains to a vulnerable population group. Specifically, at elementary schools in Chile, this percentage increases to $76.4 \%$. Given this background, this study seeks to look at preservice school teacher perception regarding their classroom practice in lower-income settings. In this qualitative study, fourteen preservice teachers were interviewed, all majoring in Education at a private university in Chile. The actual interviews were then transcribed and classified according to the corresponding themes identified in the verbatim interview transcription. The results identify two main ideas related to the tension and challenges that preservice elementary school teachers perceived during their classroom field practice with students who pertain to vulnerable population groups. Each topic is divided into four subject headings: Working in a context of violence; teacher-student relationships; official school curricula versus the executed curriculum and, the sense of learned helplessness felt among students. The second subject area refers to the challenges teachers face and is classified according to the following headings: Interaction with students, promotion of values in the classroom, managing emotions and finally, dealing with student frustration. The results of this study will, hopefully provide future teachers with a better skillset to be able to work in such challenging settings. It also serves as a precedent for teachers by improving their understanding of this phenomenon.

KeYWORDS: Primary Education, Teacher Education, Educational Environment, Vulnerable Contexts. 


\section{INTRODUCCIÓN}

En América Latina se ha diagnosticado que el 50 \% de jóvenes está en condición de vulnerabilidad, lo que también conlleva a mirar a la zona latinoamericana como un territorio de relevantes desafíos en cuanto al desarrollo (Brunner y Ganga, 2017) ${ }^{[1]}$. Actualmente, en Chile existen 1.143 .193 estudiantes que son primera prioridad de vulnerabilidad, lo que representaría a un $76,34 \%$ del total de estudiantes en el sistema escolar. En el país existe un total de 16.044 establecimientos educacionales. De este total, un $88 \%$ son de dependencia administrativa municipal o particular subvencionada, lo que presenta un Índice de Vulnerabilidad Escolar (en adelante IVE) promedio cercano al 87 \% (Junta Nacional de Auxilio, Escolar y Becas [JUNAEB], 2018).

Esta situación ha llevado a que el fenómeno de la vulnerabilidad se estudie con énfasis en la calidad de la educación, interacciones pedagógicas o en el liderazgo educacional (Maureira y Rojas, 2013; Villalta, et al., 2011). Sin embargo, una de las aristas poco investigadas es la que hace referencia a la formación inicial docente. En este sentido, resulta fundamental identificar la percepción del profesorado en formación sobre su práctica pedagógica en contextos vulnerables. Específicamente, es relevante que se identifiquen las principales tensiones, desafíos y problemáticas experimentadas, así como aquellos aspectos importantes de desarrollar y que brinden herramientas que fortalezcan el proceso de formación y les permitan desempeñarse de manera efectiva en estos contextos. La relevancia de lo anterior se debe a que la formación recibida en el pregrado para desempeñarse en estos contextos no ha sido considerada como óptima (Turra et al., 2015; Calvo, 2015), de hecho, ya a principios de la década del año 2000, existían evidencias respecto a las deficiencias en la formación inicial docente para trabajar en contextos de vulnerabilidad social/escolar (Román, 2003). Esto se relaciona directamente con otras críticas que se han hecho desde la investigación educativa y social, donde se ha destacado que el estudiantado de pedagogía recibe una precaria formación en temas de justicia social (Silva, 2017) e interculturalidad (Sanhueza, et al., 2016), elementos que se relacionan también con la multidimensionalidad de la vulnerabilidad.

En Chile, los segmentos más vulnerados son los que obtienen menores resultados en diversas evaluaciones estandarizadas (Villalta y Saavedra, 2012), lo que se evidencia en los resultados de la prueba PISA en el año 2015, donde estudiantes del cuartil más alto obtienen un $24 \%$ de mejor rendimiento en la prueba de ciencias, un $21 \%$ en lectura y un $25 \%$ en matemáticas (Organización para la Cooperación y el Desarrollo Económico [OCDE], 2016). Así, la vulnerabilidad relacionada al ámbito socioeconómico no solo impacta al tipo de escuela al que se puede acceder, sino que también al nivel de aprendizaje que se puede adquirir.

Debido a lo anterior se ha propuesto como objetivo indagar en las percepciones de docentes en formación de enseñanza primaria con respecto al trabajo educativo realizado en sus prácticas pedagógicas en contextos vulnerables. Esto cobra relevancia dado que en la literatura se ha identificado que gran parte de las personas docentes carecen de una preparación que les permita desempeñarse de manera óptima en ambientes vulnerables, ya sea porque no consideran el contexto sociocultural y habitus de sus estudiantes (Muñoz, et al., 2013; Duarte, 2003; Tenti, 2000; Dubet y Martuccelli, 1998) o porque no cuentan con las estrategias pedagógicas, psicológicas, afectivas-metodológicas necesarias para impulsar procesos de enseñanza de calidad que promuevan un aprendizaje óptimo (Oros et al., 2015).

A partir de lo anterior, es que este artículo presenta a continuación un apartado conceptual que permite instalar el concepto de vulnerabilidad de manera amplia y en particular dentro del mundo educativo; luego, se explicita la metodología utilizada, para finalmente, presentar los principales hallazgos y una discusión teórica con sus respectivas conclusiones. 


\subsection{Sobre el concepto de vulnerabilidad}

$\mathrm{Al}$ analizar el concepto de vulnerabilidad es posible develar cómo se ha ido modificando según los contextos sociales y épocas en los que se ha desplegado. Así, al revisar literatura que ha trabajado el concepto desde las ciencias sociales, se puede encontrar que a partir de la década de los setenta se intenta establecer un esfuerzo porque sea entendido desde una perspectiva más social; sin embargo, se restringe a lo socioeconómico (Fernández, 2017; Carrera, 2015; Pérez de Armiño, 2000). Esto se asienta y profundiza durante la década del ochenta, dado que la vulnerabilidad se entiende como un concepto que se relaciona con las condiciones de vida de los grupos poblacionales, lo que reduce el concepto de vulnerabilidad a una visión netamente economicista, directamente asociado con la desventaja económica (Helleiner y Frances, 1987). A finales de la década del noventa, este concepto fue abordado desde una perspectiva más crítica, en donde se da relevancia al eje del empleo y su precarización, los derechos humanos o las propias desigualdades producidas por el neoliberalismo y su forma de concebir el desarrollo (Castel, 1992). Así, se propone que son vulnerables los sectores pobres que tienen o buscan alternativas de inclusión, y los sectores medios empobrecidos que han ido perdiendo canales de inclusión (Minujín, 1999). Esto cobra absoluta relevancia si se piensa en el caso chileno, sobre todo en el marco de una transición que dio paso al libre mercado con costos humanos elevados. De acuerdo con lo anterior y ya durante la primera década del nuevo milenio, se puede apreciar que el concepto ha evolucionado hacia la complejidad, con un mayor número de factores asociados, como lo definen Cornejo et al. (2005):

Condición dinámica que resulta de la interacción de una multiplicidad de factores de riesgo y protectores, que ocurren en el ciclo vital de un sujeto y que se manifiestan en conductas o hechos de mayor o menor riesgo social, económico, psicológico, cultural, ambiental y/o biológico, produciendo una desventaja comparativa entre sujetos, familias y/o comunidades (p. 14).

Esta definición coloca al concepto de vulnerabilidad desde un lente relacional, donde la supremacía de la perspectiva económica trata de ser superada con el objetivo de complejizar y poner en dinamismo al concepto, tal como lo propone Chambers (1989) al explicitar que la vulnerabilidad no es equivalente a la pobreza, ni significa que existan carencias o necesidades, sino que a esta la componen características de riesgo, inseguridad e indefensión. Por lo tanto, para desplegar el concepto de vulnerabilidad, habría que tener en consideración el nivel microsocial (agencia de los sujetos y sus hogares), así como también elementos de nivel macro, como lo son el rol de las instituciones y distintas organizaciones (Kaztman, 2018).

En la actualidad, se propone que la vulnerabilidad permite entender la heterogeneidad dinámica de las desigualdades sociales; lo que pone en juego la relación Activos, Vulnerabilidad, Estructura de Oportunidades (Ramos, 2019). Lo anterior apunta a un análisis sobre el rol que ocupan las políticas sociales, pero también da espacio para cuestionar la gestión emancipadora de las individualidades, hogares y comunidades en el proceso de la movilidad social. Un ejemplo claro del dinamismo de este concepto se puede apreciar en el estudio de la inequidad digital, donde se establecen interesantes premisas que apuntan a una forma de vulnerabilidad de aquellas personas menos aventajadas, dado que las tecnologías y las culturas digitales también amplifican y explicitan desigualdades existentes entre clases, territorios y culturas (Claro et al., 2015).

\subsection{Sobre vulnerabilidad y escuela}

A nivel internacional existen estudios que han abordado la vulnerabilidad escolar desde diversos ángulos. Por una parte, la formación y perfeccionamiento docente juegan un rol primordial en la optimización del trabajo pedagógico en contextos vulnerables, en donde se han desarrollado programas de apoyo a profesores que hacen frente a la injusticia educativa, esto promueve procesos de enseñanza-aprendizaje de mayor calidad (Grau y García, 2017; McIntyre y Thomson, 2016; Marchant et al., 2015; Ferrada et al., 2013; Marchant et al., 2013; Sancho, Jardón y Grau, 2013). Los programas han abordado diversos ámbitos, por 
ejemplo, la autoestima de docentes y estudiantes, en donde se establece que un adecuado abordaje no solo promueve la mejora de esta en ambos grupos, sino que también mejora el rendimiento académico en áreas como Lenguaje y Matemática (Marchant et al., 2013). Otro ámbito evaluado, y ligado al anterior, es el desarrollo de competencias emocionales de directivos escolares y profesorado para la promoción de una buena autoestima de docentes y estudiantes, donde se destaca la importancia del rol que juegan ambas partes en esto (Marchant et al., 2015). La convivencia escolar también ha sido abordada como aspecto importante a trabajar en escuelas situadas en contextos vulnerables, en donde su promoción se relaciona con la disminución de actos de violencia entre estudiantes; es decir, se favorece la construcción de un ambiente que promueve la participación del alumnado y el diálogo entre sus integrantes (Grau y García, 2017). Sin embargo, también hay estudios que develan cómo las escuelas vulnerables tienen bajos y peores ambientes escolares, lo que hace que el estudiantado se sienta estigmatizado y discriminado (Ascorra et al., 2016). En esta línea, si bien se evidencian múltiples beneficios de la implementación de programas en escuelas que están insertas en contextos vulnerables, esto también ha presentado ciertos problemas, en donde docentes que han accedido a capacitaciones y tienen más años de experiencia laboral tienden colocarse en escuelas ubicadas en territorios con menor vulnerabilidad o no vulnerables, de manera que se forma una estructura donde educación y territorio promueven y reproducen condiciones de desigualdad (Mendez y Lemos 2017). En esta línea, y a modo de ejemplo, se ha estudiado la alfabetización como catalizador de reproducción de vulnerabilidad, en donde se logra ver el dinamismo de este concepto y su relación con los modelos de desarrollo, el mundo del trabajo y la estructura social, así queda en claro que la escuela y el sistema escolar operan como un reproductor de ciertas normas (Jaeger, 2017). En este sentido, la reproducción de la desigualdad es fomentada por la estructura del sistema laboral neoliberal, en donde la población estudiantil de la escuela vulnerable accede a trabajos más precarios y menos valorados.

Por otro lado, han existido estudios como el de Miranda et al. (2019), que han indagado en representaciones de personas académicas universitarias sobre la vulnerabilidad educativa, en las que se evidencia cómo se asocia directamente esta condición a conceptos como el de pobreza, riesgo y marginalidad. Aquí, nuevamente, se asocia a la población de estudiantes vulnerables con una condición socioeconómica baja y una trayectoria escolar precarizada y ligada directamente a la educación pública.

Debido a las características geopolíticas de Chile, la vulnerabilidad es una temática que ha sido ampliamente investigada durante la última década en el ámbito educacional; se han entregado múltiples evidencias que develan las fallas del modelo en cuanto a la capacidad de proporcionar justicia social e igualdad de oportunidades a la totalidad de estudiantes (Bellei, 2013; Treviño et al., 2016).

A nivel de cultura escolar, se evidencia la vulnerabilidad en su carácter más complejo, en donde son múltiples los factores presentes que intentan abordarla; de esta manera, se quita la exclusiva responsabilidad al contexto socioeconómico en que se encuentran insertas las escuelas. Algunos de los factores son, en primer lugar, la relación existente entre la cultura familiar del estudiantado vulnerable y la propia cultura escolar, que muchas veces posee un carácter antagónico que se materializa en la no consideración del habitus del alumnado, de esta manera se privilegia la estandarización de la enseñanza por parte del personal docente, la cual es encarnada a partir del currículum oficial (Muñoz et al., 2013; Tenti, 2000; Escobar, 2020). Lo anterior se contradice con la ya establecida importancia que se le debe dar al contexto y la consideración de sus particularidades al momento de diseñar el proceso de enseñanza-aprendizaje, especialmente en contextos de desventaja (Carrasco, 2008).

Un segundo factor que se debe considerar es el clima que se vive en las escuelas. Los estilos de liderazgo, la forma de trabajo existente entre docentes y la participación de madres y padres, pueden influir no solo en la formación académica de los estudiantes, sino también en aquella de carácter social. Se evidencia complejidad en la mejora de estos aspectos en aquellas escuelas en donde existe un liderazgo vertical y la visión que el personal docente posee de sus estudiantes es de desesperanza, debido a que consideran que la vulnerabilidad del alumnado y sus familias son características inherentes e imposibles de ser superadas. Por el contrario, una 
realidad distinta se evidencia en escuelas en donde existe trabajo cooperativo entre docentes, quienes creen en la superación de sus estudiantes y hay una participación activa de las familias como miembro de la comunidad educativa (Montecinos et al., 2010).

Un tercer factor que se debe considerar es la formación emocional del profesorado, en donde este presenta un dominio robusto de las dimensiones interpersonales e intrapersonales que componen la inteligencia emocional, con lo que tiende a ser muy competente al momento de ejercer (Cejudo y López, 2017); esto no resulta azaroso si se consideran investigaciones como las de Silva y Paz (2019), quienes apuntan a la necesidad de una formación inicial docente que desarrolle no solo cuestiones de carácter intelectual, sino también elementos emocionales que vayan más allá de generalidades y que logren instalarse con un foco y objetivo en la justicia social. Si bien ambas dimensiones son necesarias, estudios han evidenciado que el profesorado considera que, para un óptimo desempeño de la labor docente, son más necesarias aquellas de tipo interpersonal, por ejemplo, la habilidad de saber relacionarse y manejar las emociones de otras personas, más que las de tipo intrapersonal, materializadas, por ejemplo, en el manejo del estrés o la gestión emocional propia (Golombek y Doran, 2014). Finalmente, el tamaño del establecimiento y el rol de la familia en la escuela, encarnada en la baja participación y compromiso por las partes apoderadas (Treviño et al., 2016). Estos factores percibidos son considerados relevantes y podrían influir en el desempeño estudiantil, ya que favorece la reproducción de la vulnerabilidad.

\section{MÉTOdo}

A través de un diseño cualitativo de tipo interpretativo, la siguiente investigación busca indagar en las percepciones de docentes en formación de la carrera de Educación Básica, con respecto al trabajo educativo en contextos vulnerables.

La muestra de este estudio es de carácter no probabilístico-intencionado, en donde los criterios de selección establecidos fueron: contar con docentes en formación que, en primer lugar, hubiesen realizado al menos una práctica docente en colegios de dependencia administrativa municipal o particular subvencionada y, en segundo lugar, que el Índice de Vulnerabilidad Escolar (IVE SINAE) de estos establecimientos (centros de práctica) fuese superior al 90\%. De acuerdo con lo establecido previamente, se invitó a participar a 18 docentes en formación que cumplían con lo establecido previamente. Finalmente, 14 docentes accedieron a participar; en este grupo, 12 eran de género femenino y 2 de género masculino. Es importante mencionar que el desequilibrio en el género de la muestra podría deberse a la feminización de las carreras de educación básica en Chile. La totalidad de las personas participantes estaba matriculada en la carrera de Educación Básica de una universidad privada chilena. Al momento de la investigación, la edad promedio de la muestra era de 21 años y se encontraba cursando los dos últimos años de la carrera. Es importante mencionar que, para esta investigación, el grupo de docentes en formación fue invitado a participar de manera voluntaria, quienes accedieron, firmaron un consentimiento informado. Para asegurar el anonimato y la confidencialidad, las citas extraídas de las entrevistas se presentan con una letra E correspondiente a persona entrevistada, seguido por un número de identificación.

Los datos se recolectaron a través de una entrevista semiestructurada (Venkat et al., 2009) realizada a cada participante. El guion contaba con 16 preguntas bases, con preguntas de seguimiento para profundizar en aquellos aspectos que fuesen de interés para quien realizaba la entrevista. La duración fue entre 25 y 30 minutos, la totalidad de ellas fueron audio-grabadas y transcritas de manera literal.

Para analizar las entrevistas se realizó un análisis temático. Lo anterior con el fin de profundizar en las percepciones de las personas docentes en formación sobre las prácticas que desempeñaron en contextos vulnerables. Se siguieron los lineamientos establecidos por Braun y Clarke (2006) para la realización de este tipo de análisis; las entrevistas fueron leídas de manera reiterada para familiarizarse con ellas (primera fase), y así identificar diversos códigos que se fueron agrupando en categorías iniciales que fueran afines 
(segunda fase). Posteriormente, estos códigos categorizados permitieron que se identificaran temas generales (tercera fase), los cuales fueron revisados y refinados para corroborar que, por una parte, los códigos que los componen fuesen coherentes y, por otra, que los temas definidos fuesen diferentes entre sí (cuarta fase). Finalmente, estos temas fueron rotulados de manera simple y clara para elaborar el mapa temático (quinta fase), finalmente, fueron descritos a través de sus códigos en una narrativa que sustentara a cada uno de ellos a través de citas pertinentes extraídas de las entrevistas (sexta fase). Para establecer la confiabilidad del análisis, específicamente en la construcción de códigos, fueron dos los codificadores que analizaron las transcripciones. En primera instancia, trabajaron de manera individual, para luego comparar y estimar la concordancia de las codificaciones.

\section{Resultados}

La presente investigación buscó indagar, en las percepciones de docentes en formación de enseñanza primaria, sobre el trabajo educativo realizado durante las prácticas pedagógicas en contextos vulnerables. Del análisis realizado a las entrevistas, se identificaron dos temas principales, cada uno de ellos compuesto por cuatro códigos. La Figura 1 muestra el mapa temático elaborado a partir de los datos obtenidos.

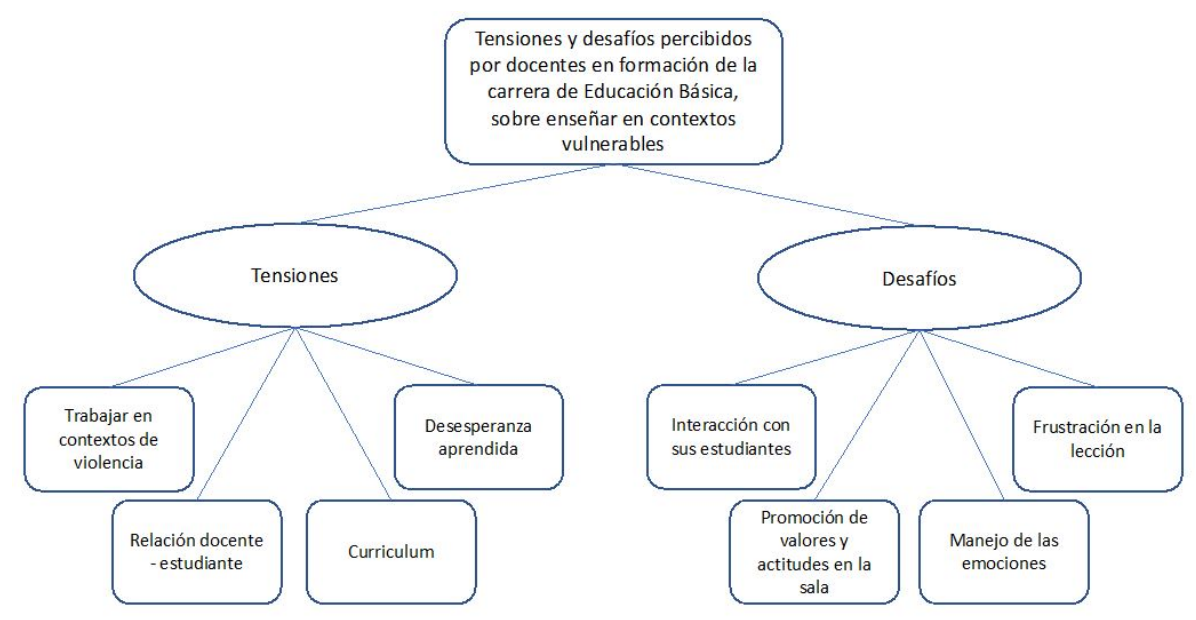

FIGURA 1.

Mapa temático compuesto por dos temas principales y sus respectivos códigos Fuente: Elaboración propia.

Dentro de las tensiones que las personas docentes en formación perciben de trabajar en contextos vulnerables, se encuentran las siguientes:

\section{Primer código: Trabajar en contextos de violencia}

El grupo de docentes en formación menciona que, en los establecimientos educativos con alto índice de vulnerabilidad, era común y recurrente ver conductas violentas en el estudiantado, las cuales se materializaban en peleas, amenazas, gritos o menoscabos. Comenta que enfrentar este tipo de situaciones era bastante complejo debido a la intensidad con la que ocurrían: "En una salida, dos alumnos se agredieron tanto verbal como físicamente, pero con palabras no adecuadas para su edad, con amenazas... fue super difícil contenerlos...” (E 14, comunicación personal, 2 de octubre de 2019).

Junto a lo anterior, las personas participantes consideran que esta violencia se encuentra naturalizada en el estudiantado. Las conversaciones que ellos y ellas tenían en diversos espacios, como el patio o dentro de la sala de clases, estaban centradas en este tópico, es decir, comentaban situaciones ocurridas durante el fin 
de semana, el día anterior o lo que escuchaban en sus barrios o de sus familiares: "los temas que los niños conversaban eran de cuarto básico y hablaban de temas de violencia, de drogas, de que le aforraron, que se fueron a cuchillazos... a las manos" (E 2, comunicación personal, 2 de octubre de 2019).

\section{Segundo código: Relación docente-estudiante}

Otra tensión señalada por las personas docentes en formación hace referencia a la relación que se establece entre el profesorado de escuelas vulnerables y sus estudiantes. Para intentar tener un dominio o manejo sobre el curso, se percibe que los profesores y las profesoras establecen estrategias basadas en el control y poder, las que mayoritariamente tienen baja efectividad con el estudiantado: "La profesora era súper estricta, de verdad me impresionó. Después entendí que era su estrategia, porque el curso era difícil. Al final siempre me decía que ni así la escuchaban o respetaban" (E10, comunicación personal, 2 de octubre de 2019).

Esta dinámica establecida por docentes de aula hace que su relación con el grupo de estudiantes sea compleja y tensa, lo que construye una imagen de la persona docente como alguien a quien el alumnado no puede acceder ni emocional ni académicamente: "hay poco respeto hacia los profesores y la relación es distante en general. Dan órdenes, pero no les explican, no van más allá...no se acercan y ellos lo necesitan” (E3, comunicación personal, 2 de octubre de 2019).

\section{Tercer código: currículum oficial versus currículum implementado}

El trabajo relacionado con el proceso de enseñanza-aprendizaje se percibe como una tensión permanente, el cual se da en los establecimientos educacionales con alto índice de vulnerabilidad. Las personas docentes en formación advierten una dualidad en el profesorado de aula, la que se materializa en cumplir con los planes establecidos para el currículum oficial versus el ritmo real con que deben abordar los objetivos para promover y alcanzar el aprendizaje en la totalidad de sus estudiantes:

La profe tiene que planificar de acuerdo con lo establecido, pero los estudiantes necesitan refuerzo y ella lo sabe... sabe que hay que ir lento, un poco para atrás para que tengan una base y no aumentar las lagunas de conocimiento que ellos tienen... el avanzar a diestra y siniestra hace que cada vez entiendan menos, que aprendan menos (E14, comunicación personal, 2 de octubre de 2019).

De lo anterior, el grupo de docentes en formación señala que visualizan, en los docentes de aula, una preocupación relacionada al nivel de logro que sus estudiantes tengan en pruebas estandarizadas, por ejemplo, el SIMCE: "El SIMCE es como un fantasma que está siempre presente en los profes, no solo en octubre.... como que todo siempre gira en torno a la prueba" (E5, comunicación personal, 2 de octubre de 2019).

\section{Cuarto código: Desesperanza aprendida}

Una cuarta tensión percibida por la muestra de docentes en formación se relaciona con la desesperanza aprendida que experimentan fuertemente sus estudiantes. En este código advierten una ausencia de proyección en el alumnado, en donde no hay mayores aspiraciones que seguir en el barrio o tener un trabajo similar al de sus progenitores o cuidadores: "Tía da lo mismo, para que vaya a estudiar si voy a estar acá siempre" (E10, comunicación personal, 2 de octubre de 2019).

Para el grupo de docentes en formación, la familia es uno de los agentes que juega un rol clave en la desesperanza aprendida que presenta la comunidad de estudiantes. Advierte que el alumnado valida los comentarios emitidos por sus familiares, por lo que cuestiona sus capacidades. Lo anterior se materializa en una limitación de los esfuerzos a nivel académico, en una disminución de la perseverancia y en un desvanecimiento de los sueños sobre el futuro: "Ninguno de los niños que yo tuve piensa en ir a la 
Universidad... dicen a mí no me exigen nada, no piensan que puedo, entonces, ¿cómo voy a ir?” (E 12, comunicación personal, 2 de octubre de 2019).

Finalmente, las personas docentes en formación presentan una sensación de impotencia frente a esta realidad; manifiestan sentirse complicadas, complicados e impotentes a la hora de enfrentar esta desesperanza en sus estudiantes:

es una sensación entre rabia, pena y angustia y yo no sé qué decirles, si lo que diga estará bien o no... no estoy preparada para esto... pero es duro escuchar a un niño chico decir: si pongo atención me va mal y si no pongo, me va igual de mal. Entonces, ¿ para qué me esfuerzo si da lo mismo? Además, en la casa ya ni me pegan si me va mal (E5, comunicación personal, 2 de octubre de 2019).

\section{SEgundo TEMA: DESAFÍOS}

\section{Primer código: Interacción con sus estudiantes}

La interacción con los y las estudiantes es identificada, por el grupo de docentes en formación, como uno de los desafíos que deben ser trabajados. A diferencia del código relación docente-estudiante, perteneciente al tema tensiones, este se centra en la relación que las futuras personas docentes establecen con sus estudiantes, la cual se percibe como compleja. El grupo menciona haber experimentado dificultades para relacionarse con el alumnado debido a que no se les considera profesores ni profesoras:

Yo creo que el dominio de grupo en estos contextos de vulnerabilidad es muy importante, uno lo intenta, pero son al final ellos los que no te ven como una profesora, te ven como una profesora practicante (...) entonces me falta manejo de grupo (E3, comunicación personal, 2 de octubre de 2019).

Para esto, las personas docentes en formación consideran que el conocimiento del contexto y de sus características es relevante. Sienten que podrán sortear de manera óptima el desafío de desempeñar la labor docente en ambientes educativos de carácter vulnerable:

uno necesita saber cómo poder trabajar con ellos, necesito herramientas para trabajar en este tipo de colegios o, por último, saber de antes como es el contexto, las necesidades de los niños y las familias, sus características emocionales ... no sé... conductuales para poder hacer bien lo que vinimos a hacer (E10, comunicación personal, 2 de octubre de 2019).

\section{Segundo código: promoción de valores y actitudes en la sala}

Las futuras personas docentes reportan que los actos violentos, de hurto y poco respeto son conductas que están presentes de manera frecuente en estos contextos. Consideran que es importante su desarrollo y promoción en el aula por parte del profesorado: "que sepan respetarse en todo sentido... desde el turno hasta a los compañeros... es difícil trabajarlo, pero tienen que aprender” (E9, comunicación personal, 2 de octubre de 2019).

Señalan que esto no debe ser realizado solo cuando ocurre un hecho puntual, sino que debe ser de manera transversal, en las distintas instancias de la jornada escolar: "siempre hay que reforzar los valores... que se cuiden, que sean buenos amigos, que sepan que los problemas se solucionan conversando y no a golpes...que sean tolerantes y no pataleteros" (E2, comunicación personal, 2 de octubre de 2019). 


\section{Tercer código: manejo de las emociones}

El grupo de docentes en formación advierte dificultades para poder comprender las historias de vida de sus estudiantes. Declaran sentirse afectadas y afectados, no solo dentro de la escuela al momento de su práctica, sino también una vez que están fuera de ella: "Tengo que saber manejar lo emocional, enfrentarme a esto bien... como que llego a mi casa y me pongo a llorar porque me da pena lo que viven... su realidad... y no sé qué hacer... qué decirles" (E8, comunicación personal, 2 de octubre de 2019).

Advierten la necesidad de contar con herramientas que les ayude a poder manejar apropiadamente sus emociones, para poder ser un apoyo para sus estudiantes; sin embargo, la profesora o el profesor de aula les brindaba apoyo para enfrentar estas situaciones:

no sé cómo, esto no me lo han enseñado... la profesora jefa me decía... tú no puedes llorar, no puedes salir llorando todos los días de práctica... tienes que entender que no les puedes solucionar la vida, pero síles puedes entregar herramientas para mejorar... y eso me daba ánimos (E1, comunicación personal, 2 de octubre de 2019).

\section{Cuarto código: Frustración en la lección}

Con respecto al proceso de enseñanza, la muestra de docentes en formación declara experimentar dificultades al momento de realizar sus clases. Señala que uno de los aspectos más complejos tiene relación con la motivación de los y las estudiantes, ya que era difícil que participaran y trabajaran en clases, independiente de las estrategias que se utilizarán para lograr este fin:

yo preparaba harto mis clases, pero ellos (los estudiantes) solo pensaban en irse, no escuchaban la clase... hacía los juegos de mímica, pero ni eso resultaba, estaban muy ansiosos de irse...y de ahí ya recurría al clásico shhh o hablar más fuerte, pero tampoco resultaba (E 13, comunicación personal, 2 de octubre de 2019).

Otro aspecto complejo advertido por las personas docentes en formación se relacionaba con las metodologías de enseñanza que utilizaban en sus prácticas educativas. Mencionan que llegaban con ideas innovadoras y clases diseñadas con uso de materiales que promovieran una mejor comprensión de lo trabajado, pero que, sin embargo, el profesorado de aula no les permitía realizarlas debido a la cantidad de tiempo que utilizaban:

Se tiende a mecanizar mucho la enseñanza para poder cumplir los objetivos, entonces no me dejaban usar material concreto para la suma porque se gasta tiempo, es más lento según la profe... como que al final me frenaban las ideas (E7, comunicación personal, 2 de octubre de 2019).

\section{Discusión y Conclusiones}

La presente investigación buscó indagar, en las percepciones de docentes en formación de la carrera de Educación Básica, sobre el trabajo educativo en contextos vulnerables.

De acuerdo con los resultados establecidos, se evidencian tensiones y desafíos percibidos por las personas docentes en formación durante la realización de sus prácticas pedagógicas, las cuales se realizaron en establecimientos que presentan un alto índice de vulnerabilidad.

Dentro de las tensiones, el grupo de docentes en formación señaló la constante preocupación que experimenta el profesorado por cumplir con lo establecido en el currículum oficial; declaran estar conscientes de que sus estudiantes tienen otros ritmos y niveles de aprendizaje que podrían diferir de lo establecido. Esto se condice con lo señalado por Muñoz et al. (2013) y Carrasco (2008) sobre la importancia de considerar el contexto para el proceso de enseñanza-aprendizaje. Otra tensión identificada es la desesperanza aprendida que las personas docentes en formación percibieron en sus estudiantes, quienes ven limitadas o anuladas sus 
proyecciones futuras; lo anterior podría estar relacionado a la baja motivación que presentan en la lección. Por tanto, sería importante desarrollar y promover una buena autoestima en el estudiantado, tal como lo establecen Marchant et al. (2013; 2015). El liderazgo autoritario y vertical de personal docente de aula también se encuentra presente en estos contextos, por lo que es fundamental abordarlos para promover un proceso de enseñanza-aprendizaje de calidad, tal como lo establecen Montecinos et al. (2010), quienes destacan que la integración de la familia como agente activo de la comunidad educativa, el trabajo cooperativo entre el profesorado y el que los docentes crean en sus estudiantes, son aspectos claves que promoverán la mejora escolar en estos contextos, al transitar de un liderazgo vertical a uno más horizontal y democrático.

Por otra parte, los resultados identificaron desafíos percibidos por el grupo de docentes en formación que realizó sus prácticas en contextos vulnerables. Una de las personas participantes se centró en la importancia de la promoción de valores y actitudes en la sala de clase. Para esto sería interesante tener presente lo trabajado por Grau y García (2017), quienes, a través de una capacitación en convivencia escolar, promovieron un ambiente que favoreció el diálogo y participación del estudiantado. Otro desafío advertido por el profesorado es la importancia que le otorgan a la formación en emociones, lo cual no se condice con lo establecido por Golombek y Doran (2014), quienes relegan la gestión emocional propia a un segundo plano, debido a que consideran que, en primer lugar, se deben trabajar las emociones de las otras personas. Frente a la importancia de la formación emocional, el grupo de docentes en formación percibió que esto le brindará herramientas para trabajar de mejor manera con sus estudiantes, pues le entrega un apoyo más efectivo, tal como lo señalan Cejudo y López (2017).

De lo anterior se puede concluir que la persona docente es un agente clave y determinante en el proceso de aprendizaje de sus estudiantes en estos contextos, por lo que requiere de herramientas que le permitan desenvolverse de manera óptima en el proceso de enseñanza-aprendizaje. Estas herramientas se relacionan con su formación emocional, cómo enfrenta incidentes críticos, la forma de abordar el currículum y, especialmente, la interacción que establece con sus estudiantes. Lo anterior hace que se deba reconsiderar la caracterización de la vulnerabilidad. Esto, porque generalmente se ha centrado en el ámbito socioeconómico o en la precaria calidad de educación recibida, ya que existen otros factores que le entregan una perspectiva más compleja y dinámica.

Lo anterior, requeriría de una formación docente que profundice e instale de manera transversal cuestiones sobre la justicia social (Silva, 2017), el trabajo multi e intercultural (Sanhueza et al., 2016) y, por sobre todo, una preparación en cuanto al trabajo emocional (Silva y Paz, 2019) de lo que significa enfrentarse a otros que viven en contextos de riesgo, incertidumbre e inseguridad. En este sentido, es recomendable que la formación del profesorado permita abrir y democratizar los espacios escolares, en donde las relaciones y vínculos sociales se abran para permitir y fortalecer el trabajo intergeneracional y democrático entre el personal directivo, docentes, partes apoderadas y estudiantes (Duarte, 2003; Escobar, 2020).

En resumen, es importante mencionar que la presente investigación es relevante debido a que establece aspectos necesarios, que se deben tener en consideración al momento de planificar y realizar prácticas educativas en establecimientos que tienen un alto índice de vulnerabilidad. Lo anterior hace que lo acá establecido sirva de insumo a los programas de formación inicial docente, para fortalecer este proceso a través de la entrega de herramientas adecuadas, que le permita al futuro profesorado desempeñarse de manera óptima en escuelas vulnerables, al promover en sus estudiantes un proceso de aprendizaje efectivo. A la vez, es importante que docentes en formación conozcan y comprendan la complejidad de la vulnerabilidad, por medio de otras variables que inciden en ésta, más allá de lo socioeconómico, por ejemplo, aquellas de carácter territorial o cultural que profundizan en la comprensión de este fenómeno. A la vez, se espera que oriente el trabajo del cuerpo docente que se desempeña en estos contextos.

Una de las principales limitaciones que posee esta investigación tiene relación con las personas participantes, quienes pertenecen en su totalidad a un mismo programa de formación inicial docente. De esto se desprende, como proyección, indagar en las experiencias de práctica en contextos vulnerables de 
docentes en formación que pertenezcan a programas de pedagogía de diversas instituciones de Educación Superior. Otra proyección sería indagar en la migración que experimenta el profesorado que trabaja en escuelas vulnerables a aquellas que lo hacen en territorios más favorecidos.

\section{Referencias Bibliográficas}

Ascorra, P., López, V., Núñez, C. G., Bilbao, M. A., Gómez, G. y Morales, M. (2016). Relación entre segregación y convivencia escolar en escuelas chilenas con altos puntajes PISA 2009. Universitas Psychologica, 15(1), 65-78. h ttps://doi.org/10.11144/Javeriana.upsy15-1.rsce

Bellei, C. (2013). El estudio de la segregación socioeconómica y académica de la educación chilena. Estudiospedagógicos, 39(1), 325-345. https://doi.org/10.4067/S0718-07052013000100019

Braun, V. y Clarke, V. (2006). Using thematic analysis in psychology. Qualitative Research in Psychology, 3(2), 77-101. https://doi.org/10.1191/1478088706qp063oa

Brunner, J.J. y Ganga, F. (2017). Vulnerabilidad educacional en América Latina: Una aproximación desde la sociología de la educación con foco en la educación temprana. Revista Opción, 33(84), 12-37. https://www.redalyc.org/p $\mathrm{df} / 310 / 31054991002 . \mathrm{pdf}$

Calvo, G. (2015). La formación de docentes para la inclusión educativa. Páginas De Educación, 6(1), 19-35. http://w ww.scielo.edu.uy/pdf/pe/v6n1/v6n1a02.pdf

Carrasco, A. (2008). Investigación en efectividad y mejora escolar ¿Nueva agenda? Revista Iberoamericana sobre Calidad, Eficaciay Cambio en Educación (REICE), 6(4), 5-23. https://www.redalyc.org/pdf/551/55160402.pdf

Carrera, C. A. (2015). La vulnerabilidad de lo social: una mirada a tres discursos sobre lo 'vulnerable'. Revista Trabajo Social, (10), 171-188. https://revistas.udea.edu.co/index.php/revistraso/article/view/23832/19557

Castel, R. (1992). La inserción y los nuevos retos de las intervenciones sociales. En F. Álvarez-Uría (Comp.). Marginación e inserción. Los nuevos retos de las politicas sociales. (pp. 25-36). Madrid: Endymión.

Cejudo, J. y López, M. (2017). Importancia de la inteligencia emocional en la práctica docente: un estudio con maestros. Psicologia Educativa, 23(1), 29-36. https://doi.org/10.1016/j.pse.2016.11.001

Chambers, R. (1989). Editorial Introduction: Vulnerability, Coping and Policy. IDS Bulletin, 20, 1-7. https://doi.o $\mathrm{rg} / 10.1111 / \mathrm{j} .1759-5436.1989 . \mathrm{mp20002001.x}$

Claro, M., Cabello, T., San Martín, E. y Nussbaum, M. (2015). Comparing marginal effects of Chilean students' economic, social and cultural status on digital versus reading and mathematics performance. Computers $y$ Education, 82, 1-10. https://doi.org/10.1016/j.compedu.2014.10.018

Cornejo, A., Céspedes, P., Escobar, D., Núñez, R., Reyes, G. y Rojas, K. (2005). SINAE Sistema Nacional de Asignación con Equidad para Becas JUNAEB: Una nueva visión en la construcción de igualdad de oportunidades en la infancia. Gobierno de Chile, Junta Nacional de Auxilio Escolar y Becas.

Duarte, K. (2003). Mundos jóvenes, mundos adultos: lo generacional y la reconstrucción de los puentes rotos en el Liceo. Última década, 10(16), 95-113. https://doi.org/10.4067/S0718-22362002000100004

Dubet, F. y Martuccelli, D. (1998). En La Escuela Sociología de la experiencia escolar. Losada.

Escobar, S. (2020). Las relaciones de poder desde la perspectiva juvenil: Continuidades y rupturas con el liceo. Revista de Ciencias Sociales, 26, 190-205. https://doi.org/10.31876/rcs.v26i0.34122

Fernández, J. (2017). Alumnado inmigrante en la ESO: Vulnerabilidad pedagógica del sistema educativo. Educación XX1, 20(1), 121-140. https://doi.org/10.5944/educxx1.17494

Ferrada, D., Turra, O. y Villena, A. (2013). Currículum transformador de educación inicial para profesores en contexto de vulnerabilidad social. Cuadernos de Pesquisa, 43(149), 642-661. http://dx.doi.org/10.1590/S0100-157420 13000200013

Junta Nacional de Auxilio, Escolar y Becas [JUNAEB]. (2018). IVE, JUNAEB. Ministerio de Educación. https://w ww.junaeb.cl/ive 
Golombek, P. y Doran, M. (2014). Unifying cognition, emotion and activity in language teacher professional development. Teaching and Teacher Education, 39, 102-111. https://doi.org/10.1016/j.tate.2014.01.002

Grau, R. y García, L. (2017). Learning to live together: a challenge for schools located in contexts of social vulnerability. Journal of Peace Education, 14(2), 137-154. https://doi.org/10.1080/17400201.2017.1291417

Helleiner, G. y Frances, S. (1987). El sistema internacional y la protección de los grupos vulnerables. En G. Cornia, G., R. Jolly y F. Stewart (Eds.), Ajuste con rostro humano. (pp. 163-193).Vol.1. Siglo Veintiuno Editores.

Jaeger, E. (2017). Reproducing vulnerability: a Bourdieuian analysis of readers who struggle in neoliberal times. British Journal of Sociology in Education, 38(7), 975-990. https://doi.org/10.1080/01425692.2016.1213158

Kaztman, R. (2018). Capacitación en Enfoque sobre Activos, Vulnerabilidad y Estructura de Oportunidades AVEO. FLACSO Cuba, Universidad de La Habana, Fundación Friedrich Ebert.

Maureira, O. y Rojas, A. (2013). Características del Liderazgo Transformacional en Docentes de Lenguaje y Comunicación en una Muestra de Establecimientos Escolares de Vulnerabilidad Social. Estudios pedagógicos, 39(1), 115-127. https://doi.org/10.4067/S0718-07052013000100007

Marchant, T., Milicic, N. y Álamos, P. (2013). Impacto en los niños de un programa de desarrollo socio-emocional en dos colegios vulnerables en Chile. Revista Iberoamericana de Evaluación Educativa, 6(2), 167-187. https://r epositorio.uc.cl/handle/11534/37975

Marchant, T., Milicic, N. y Álamos, P. (2015). Competencias socioemocionales: capacitación de directivos y docentes y su impacto en la autoestima de alumnos de $3^{\circ}$ a $7^{\circ}$ básico. Revista Iberoamericana de Evaluación Educativa, $8(2), 203-218$. https://revistas.uam.es/riee/article/view/2885

McIntyre, J. y Thomson, P. (2016). Poverty, schooling and beginning teachers who make a difference: a case study from England. En J. Lampert y B. Burnet (Eds.), Teacher Education for High Poverty Schools. (pp.153-170). Springer.

Mendez, V.y Lemos, C. (2017). Desigualdade escolar e vulnerabilidade social no territorio. Educar em Revista, 33(2), 71-87. https://doi.org/10.1590/0104-4060.51372

Minujín, A. (1999). ¿La gran exclusión? Vulnerabilidad y exclusión en América Latina, en D. Filmus (Ed.) Los noventa. Politica, sociedad y cultura en América Latina y Argentina del fin de siglo. (pp. 53-77). Flacso / Eudeba.

Miranda, J., Valenzuela, J., Jurado de los Santos, P. y Reyes, M. (2019). Representaciones docentes en torno al concepto de vulnerabilidad educativa en el contexto de Educación Superior en Chile. Revista Espacios, 40(36), 14-30. htt ps://www.revistaespacios.com/a19v40n36/a19v40n36p14.pdf

Montecinos, C., Sisto, V. y Ahumada, L. (2010). The construction of parents and teachers as agents for the improvement of municipal schools in Chile. Comparative Education, 46(4), 487-508. https://doi.org/10.1080 /03050068.2010.519481

Muñoz, C., Ajagan, L., Sáez, G., Cea, R. y Luengo, H. (2013). Relaciones dialécticas antagónicas entre la cultura escolar y la cultura familiar de niños y niñas de contextos vulnerables. Universum, 28(1), 129-148. https://doi.org/10 .4067/S0718-23762013000100007

Organización para la Cooperación y Desarrollo Económico [OCDE]. (2016). PISA 2015: Resultados Claves. OECD Publishing.

Oros, L., Menghi, M., Richaud, M. y Ghiglione, M. (2015). Educar en medios socialmente vulnerables: el valor de la capacitación docente. Contextos Educativos, 18, 79-92. https://doi.org/10.18172/con.2626

Pérez de Armiño, K. (2000). Diccionario de Acción Humanitaria y Cooperación al Desarrollo. Icaria.

Ramos, D. (2019). Entendiendo la vulnerabilidad social: una mirada desde sus principales teóricos. Revista Estudios del Desarrollo Social: Cuba y América Latina, 7(1), 139-154. http://scielo.sld.cu/pdf/reds/v7n1/2308-0132-re ds-7-01-139.pdf

Román, C.M. (2003). ¿Por qué los docentes no pueden desarrollar procesos de enseñanza aprendizaje de calidad en contextos sociales vulnerables? ILADES.

Sancho, C., Jardón, P. y Grau, R. (2013). Formación y actualización pedagógica del profesorado como facilitadores de la educación inclusiva: una base de datos inclusiva en red. Revista Nacional e Internacional de Educación Inclusiva, 6(3), 134-149. https://revistaeducacioninclusiva.es/index.php/REI/article/view/156 
Sanhueza, S., Patrick, P., Chuan-Chih, H., Domínguez, J., Friz, M. y Quintriqueo, S. (2016). Competencias Comunicativas Interculturales en la formación inicial docente: El caso de tres universidades regionales de Chile. Estudios pedagógicos (Valdivia), 42(4), 183-200. https://doi.org/10.4067/S0718-07052016000500011

Silva, I. (2017). Formación docente para la justicia social en un Chile desigual. En I. Silva, J. Diniz y K. Zeichner, Justicia Social. La dimensión olvidada de la formación docente. (pp.121-144). Mutante Editores.

Silva, I. y Paz, E. (2019). Una reflexión acerca de la indagación narrativa autobiográfica en formadores/as de docentes para la justicia social. Perspectiva Educacional, 58(2), 169-189. https://doi.org/10.4151/07189729-Vol.58-Iss. 2-Art.953

Tenti, E. (2000). Culturas Juveniles y Cultura Escolar. UNESCO.

Treviño, E., Valenzuela, J. P. y Villalobos, C. (2016). Whithin-school segregation in Chilean school system: What factors explain it? How efficient is this practice for fostering student achievement and equaty? Learning and individual differences, 51, 365-371. https://doi.org/10.1016/j.lindif.2016.08.021

Turra, O., Valdebenito, V. y Torres, A. (2015). Teaching competencies for performing in school vulnerability contexts in Chile. Procedia - Social and Behavioral Sciences, 197, 1236-1241. https://doi.org/10.1016/j.sbspro.2015.07 .386

Venkat, H., Osman, R. y Booth, S. (2009). Diversity in pre-service teachers' understandings of research. Education as Change, 13(2), 245-261. https://doi.org/10.1080/16823200903234653

Villalta, M., Martinic, S. y Guzmán, M. (2011). Elementos de la interacción didáctica en la sala de clase que contribuyen al aprendizaje en contexto social vulnerable. Revista Mexicana de Investigación Educativa, 16(51), 1137-1158. h ttps://www.redalyc.org/pdf/140/14019203006.pdf

Villalta, M. y Saavedra, E. (2012). Cultura escolar, prácticas de enseñanza y resiliencia en alumnos y profesores de contextos sociales vulnerables. Universitas Psychologica, 11(1), 67-78. https://doi.org/10.11144/Javeriana.upsy 11-1.cepe

\section{Notas}

[1] Este estudio declara la información en base a resultados de pruebas cognitivas PISA, en donde no participaron la totalidad de los países de Latinoamérica.

\section{INFORMACIÓN ADICIONAL}

Cómo citar: Yancovic-Allen, M. y Escobar-González, S. (2022). Percepciones de docentes en formación de pedagogía básica sobre educar en contextos vulnerables. Revista Educación, 46(1). http://doi.org/10.15517 /revedu.v46i1.43787 